[a042]

\title{
Crystal structure of $\mathrm{H}_{4} \mathrm{~L}^{1}\left(N-N^{\prime}\right.$-Bis(o- hydroxybenzoyl)ethylenediamine)
}

\author{
M. A. Vázquez-Fernández, M. I. Fernández, B. Fernández, A. M González-Noya, M. J. Rodríguez-Doutón
}

Dpto. de Química Inorgánica. Facultad de Ciencias. Univ. de Santiago de Compostela. Lugo 27002 (Spain).

\begin{abstract}
Three bis-amide bis-phenoxy $\mathrm{N}_{2} \mathrm{O}_{2}$ ligands were obtained from the 2:1 molar reaction of phenyl salicylate and the diamines (1,2diaminoethane, 1,3-diaminopropane and 1,4-diaminobutane) to yield $\mathrm{H}_{4} \mathrm{~L}^{\mathrm{n}}\left(\mathrm{H}_{4} \mathrm{~L}^{1}, \mathrm{H}_{4} \mathrm{~L}^{2}, \mathrm{H}_{4} \mathrm{~L}^{3}\right.$ respectively). The ligands have been characterised by elemental analysis, IR, and ${ }^{1} \mathrm{H}{ }^{13} \mathrm{C}$ NMR spectroscopies, mass spectrometry (ES) and X-ray diffraction.

\section{INTRODUCTION}

Our interest in this kind of ligands derives from the known ability of ligands containing amide groups to stabilise high oxidation states of metal ions when coordinated with the deprotonated nitrogen atom.

We have thought that the utilization of tetraanionic bis-amide bis-phenoxy ligands should favour the aggregation of neighbouring complexes through these donors atoms ${ }^{1}$ (amide and phenoxo oxygen). In previous papers we reported the synthesis, structures and properties of $\mathrm{Mn}$ (III) complexes with asymmetrical trianionic amido-imino-phenoxo ligands. At this goal we have designed the tetraanionic ligands, $\mathrm{H}_{4} \mathrm{~L}^{\mathrm{n}}$ that contain six potential donor atoms: two amide nitrogen, two phenoxo and two amide oxygen atoms.
\end{abstract}

\section{RESULTS AND DISCUSSION}

$\mathrm{H}_{4} \mathrm{~L}^{\mathrm{n}}$ were prepared according to the literature ${ }^{2}$, in this case by a 2:1 molar reaction of phenyl salicylate and the diamines with methanol at $180-190{ }^{\circ} \mathrm{C}$ for $1 \mathrm{~h}$. The product was treated with diethyl ether to obtain a white powder at almost quantitative yield. White crystals of $\mathrm{H}_{4} \mathrm{~L}^{10}$, suitable for single crystal X-ray diffraction studies, were obtained by slow evaporation of methanol solution of the ligand. The characteristiques of the ligands are :

Ligand $\mathrm{H}_{4} \mathrm{~L}^{1}$ : M.p. $180{ }^{\circ} \mathrm{C}$. Anal. Calc. for $\mathrm{C}_{16} \mathrm{H}_{16} \mathrm{~N}_{2} \mathrm{O}_{4}$ : C, 64.0; H, 5.4; N, 9.3. Found: C,64.0; H, 5.5; N,9.4\%. MS ES (m/z): 301; IR (KBr, cm ${ }^{-1}$ ): $v(\mathrm{~N}-\mathrm{H}) 3407, v(\mathrm{O}-\mathrm{H}) 3060, v$ (amido I) 1642, v(amido II) 1550, v(C-O) $1254 .{ }^{1} \mathrm{H}$ NMR (DMSO-d ${ }^{6}$, ppm): $\delta$ 8.87 (s, 2H), 6.83-6.88 (m, 4H), 7.37 (t, 2H), 7.78 (d, 2H), 3.48 (d, 2H). ${ }^{13} \mathrm{C}$ NMR (DMSO-d $\left.{ }^{6}, \mathrm{ppm}\right): \delta 160.7$ (C-OH), 170.0 $(\mathrm{C}=\mathrm{O}), 39.3\left(\mathrm{CH}_{2}\right)$.

Ligand $\mathrm{H}_{4} \mathrm{~L}^{2}$ : M.p. $177^{\circ} \mathrm{C}$. Anal Calc. for $\mathrm{C}_{17} \mathrm{H}_{18} \mathrm{~N}_{2} \mathrm{O}_{4}$ : C,64.9; H, 5.7; N, 8.9. Found: C,63.8; H, 6.1; N,9.1\%. MS ES (m/z): 315; IR (KBr, cm $\left.{ }^{-1}\right): v(\mathrm{~N}-\mathrm{H}) 3377, v(\mathrm{O}-\mathrm{H}) 3077, v$ (amido I) 1645, v(amido II) 1545, v(C-O) 1246. ${ }^{1} \mathrm{H}$ NMR (DMSO-d $\left.{ }^{6}, \mathrm{ppm}\right): \delta 8.81$ (s, 2H), 6.83-6.88 (m, 4H), 7.37 (t, 2H), $7.80(\mathrm{~d}, 2 \mathrm{H}), 3.34-3.48(\mathrm{~m}, 4 \mathrm{H}), 1.82$ (q, 2H). ${ }^{13} \mathrm{C}$ NMR (DMSO-d $\left.{ }^{6}, \mathrm{ppm}\right): \delta 161.3(\mathrm{C}-$ $\mathrm{OH}), 169.7(\mathrm{C}=\mathrm{O}), 37.4\left(\mathrm{CH}_{2}\right), 29.6\left(\mathrm{CH}_{2}\right)$.

Ligand $\mathrm{H}_{4} \mathrm{~L}^{3}$ : M.p. $127^{\circ} \mathrm{C}$. Anal Calc. for $\mathrm{C}_{18} \mathrm{H}_{20} \mathrm{~N}_{2} \mathrm{O}_{4}$ : C,65.8; H, 6.1; N, 8.5. Found: C,66.2; H, 5.6; N,8.6\%. MS ES (m/z): 329; IR (KBr, cm $\left.{ }^{-1}\right): v(\mathrm{~N}-\mathrm{H}) 3407, v(\mathrm{O}-\mathrm{H}) 3057, v$ (amido I) 1643, v(amido II) 1586, v(C-O) 1259. ${ }^{1} \mathrm{H}$ NMR (DMSO-d $\left.{ }^{6}, \mathrm{ppm}\right): \delta 9.17$ (s, 2H), 7.20-7.80 (m, 4H), 3.34-3.28 (m, 4H), 1.55 (m, 2H). ${ }^{13}$ C NMR (DMSO-d $\left.{ }^{6}, \mathrm{ppm}\right): \delta 133.9(\mathrm{C}-\mathrm{OH}), 161.6$ (C=O), 27.5 
$\left(\mathrm{CH}_{2}\right), 39.0\left(\mathrm{CH}_{2}\right)$.

Crystal structure, with the numbering scheme, is shown in figure 1. Selected bond lengths and angles, as well as potential hydrogen bonds and bonding scheme, are listed in table 1 .

The crystal structure reveals that $\mathrm{H}_{4} \mathrm{~L}$ exists as discrete molecules. The $\mathrm{C}(14)-\mathrm{O}(15)$ and $\mathrm{C}(8)-\mathrm{O}(9)$ distances of $1,257 \mathrm{y}$ $1,260 \AA$ respectively, are consistent with. $\mathrm{C}=\mathrm{O}$ double bonding of amide groups. The two oxygen $\mathrm{O}(1)$ and $\mathrm{O}(22)$ atoms are forming phenolic groups, and they present C-O distances $\mathrm{O}(1)-\mathrm{C}(2)$ de $1,360(4)$ and $\mathrm{O}(22)-\mathrm{C}(21)$ of $1,351(4) \AA$, corresponding to the expected single bonds. The distance $\mathrm{O}(1)-\mathrm{H}(1)$ de 0,99(4) $\AA$ is longer than distance $\mathrm{O}(22)-\mathrm{H}(22)$ of 0,91(3) $\AA$.

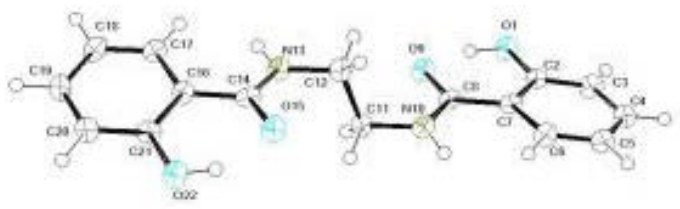

Figure 1. Crystal structure of $\mathrm{H}_{4} \mathrm{~L}^{1}$.

\section{3d structure from X-ray data ${ }^{3}$}

Intermolecular hydrogen bonds exist between nitrogen atoms $\mathrm{N}(10)$ and $\mathrm{N}(13)$ and their neighbouring phenolic oxygen atoms $\mathrm{O}(1)$ and $\mathrm{O}(22)$. Other intramolecular hydrogen bonds exists between the phenolic oxygen atoms $\mathrm{O}(1)$ and $\mathrm{O}(9)$, $\mathrm{O}(22)-$ $\mathrm{O}(15)$ and $\mathrm{O}(22)-\mathrm{O}(15)$ (see table 2 and figure 2).

Table1: Selected bond lengths $(\AA)$ and angles $\left(^{\circ}\right)$ for $\mathrm{H}_{4} \mathrm{~L}^{1}$.

\begin{tabular}{llll}
\hline $\mathrm{O}(1)-\mathrm{C}(2)$ & $1,360(4)$ & $\mathrm{O}(22)-\mathrm{C}(21)$ & $1,351(4)$ \\
$\mathrm{O}(1)-\mathrm{H}(1)$ & $0,99(4)$ & $\mathrm{O}(22)-\mathrm{H}(22)$ & $0,91(3)$ \\
$\mathrm{C}(8)-\mathrm{O}(9)$ & $1,260(4)$ & $\mathrm{C}(14)-\mathrm{O}(15)$ & $1,257(4)$ \\
$\mathrm{C}(8)-\mathrm{N}(10)$ & $1,342(5)$ & $\mathrm{C}(14)-\mathrm{N}(13)$ & $1,340(4)$ \\
$\mathrm{N}(10)-\mathrm{C}(11)$ & $1,455(5)$ & $\mathrm{N}(13)-\mathrm{C}(12)$ & $1,449(4)$ \\
$\mathrm{N}(10)-\mathrm{H}(10)$ & $0,90(4)$ & $\mathrm{N}(13)-\mathrm{H}(13)$ & $0,89(4)$ \\
$\mathrm{C}(11)-\mathrm{C}(12)$ & $1,523(5)$ & & \\
& & & $106(3)$ \\
$\mathrm{C}(2)-\mathrm{O}(1)-\mathrm{H}(1)$ & $103(3)$ & $\mathrm{C}(21)-\mathrm{O}(22)-\mathrm{H}(22)$ & $119,4(3)$ \\
$\mathrm{O}(1)-\mathrm{C}(2)-\mathrm{C}(3)$ & $119,3(3)$ & $\mathrm{O}(22)-\mathrm{C}(21)-\mathrm{C}(20)$ & $122,7(3)$ \\
$\mathrm{C}(6)-\mathrm{C}(7)-\mathrm{C}(8)$ & $123,4(3)$ & $\mathrm{C}(17)-\mathrm{C}(16)-\mathrm{C}(14)$ & $122,7(3)$ \\
$\mathrm{C}(8)-\mathrm{N}(10)-\mathrm{C}(7)$ & $119,3(3)$ & $\mathrm{C}(14)-\mathrm{N}(13)-\mathrm{C}(12)$ & $111,7(3)$ \\
$\mathrm{N}(10)-\mathrm{C}(11)-\mathrm{C}(12)$ & $112,2(3)$ & $\mathrm{N}(13)-\mathrm{C}(12)-\mathrm{C}(11)$ & \\
\hline
\end{tabular}

Table2: Hydrogen bonds

\begin{tabular}{|c|c|c|c|c|}
\hline D-H...A & $\mathrm{d}(\mathrm{D}-\mathrm{H})$ & d(H...A) & $d(D \ldots A)$ & (DHA) \\
\hline $\mathrm{N}(10)--\mathrm{H}(10) \quad . . \mathrm{O}(1)^{* *}$ & $0.90(4)$ & $2.13(4)$ & $2.952(4)$ & 152(3) \\
\hline $\mathrm{N}(13)--\mathrm{H}(13) \quad . . \mathrm{O}(22)^{*}$ & $0.90(3)$ & $2.13(4)$ & $2.926(4)$ & $147(3)$ \\
\hline Intra $1 \mathrm{O}(1) \quad--\mathrm{H}(1) \quad . . \mathrm{O}(9)$ & $0.99(3)$ & $1.57(3)$ & 2.502(3) & 156(3) \\
\hline Intra $\mathrm{O}(22)--\mathrm{H}(22) \quad . . \mathrm{O}(15)$ & $0.91(3)$ & $1.67(4)$ & $2.504(3)$ & 152(3) \\
\hline Intra $\mathrm{O}(22)--\mathrm{H}(22) \quad . . \mathrm{O}(15)$ & $0.94(4)$ & $2.60(3)$ & $2.910(4)$ & $100(2)$ \\
\hline
\end{tabular}

symmetry operations $*=-1+\mathrm{x}, \mathrm{y}, \mathrm{z}, \quad * *=1+\mathrm{x}, \mathrm{y}, \mathrm{z}$

\section{REFERENCES}


1. (a) M. R. Bermejo, A. M. González Noya, V. Abad, M. I. Fernández, M. Maneiro, R. Pedrido, M. Vázquez; Eur. J. Inorg. Chem, 2004, 18, 3696; (b) J.-P. Costes, F. Dahan, B. Donnadieu, M. J. Rodríguez-Doutón, M. I. Fernández García, A.

Bousseksou, J.-P. Tuchages; Inorg. Chem. 2004, 43(8), 2736-2744.

2. H. Ojima, K. Aichi, Nippon Kagaku Kaishi, 1967, 88 (3), 329-333.

3. Left button of mouse to move the molecule, right button to display an options menu. 